\title{
Georg Forsters Entwurf einer „Wissenschaft vom Menschen"
}

\author{
Hans Erich Bödeker
}

\author{
Georg Forster's Outline of a "Science of Man"
}

The major focus of the article is on Georg Forster's mode of elaborating a "science of man" in its theoretical and cultural contexts. The study aims at identifying Forster's distinct interests in the specificity of mankind and his interpretation of both the reasons for its diversity and its different stages of development. Forster, the article argues, used a historicized version of Enlightenment natural history in order to analyse man as a natural as well as a cultural being. At the same time, put anachronistically, Forster constituted the reciprocity of physical and cultural anthropology. However, he differs from Enlightenment historical thinking in that he interprets history as a contingency. Finally, the article maintains that Forster deliberately conceived of the "science of man" as a multidisciplinary empirical science.

Keywords: science of man, natural history, anthropology, ethnology, history, empirical research

Schüsse/wörter: Wissenschaft vom Menschen, Naturgeschichte, Anthropologie, Ethnologie, Geschichte, Empirische Forschung

Für Wolfgang Proß

Der weitgereiste Naturforscher Georg Forster hat seine Erkenntnisinteressen wiederholt formuliert (vgl. Uhlig 2004). 1789 bringt er sie prägnant auf den Begriff:

Dem Menschen liegt unstreitig kein Gegenstand näher als der Mensch selbst in allen seinen mannichfaltigen Verhältnissen der Gestalt, der Entwicklung, der Verfassung, der Zeit und des Ortes. Die Vergleichung unzähliger Abweichungen von unserer Lebensweise, die Betrachtung dessen, was in diesen verschiedenen Gemälden auf unsern eignen Zustand anwendbar ist, die Erweiterung einer Menge von Ideen, Vorstellungen, Begriffen und Neigungen, die bereits in uns vorhanden waren, aber durch ähnliche oder auch entgegengesetzte Züge im 
Charakter verschiedener Nationen erst angestoßen wurden, sind eben so viele kräftige Mittel die Aufmerksamkeit des Verstandes zu fesseln. (AA, V: 278) ${ }^{1}$

Weniger der einzelne Mensch als vielmehr die Menschheit in der Mannigfaltigkeit ihrer Erscheinungen stand im Zentrum seines Denkens. Dieses Erkenntnisinteresse spiegelt die Fremderfahrung wider, die Forster während seiner Teilnahme an der zweiten Entdeckungsreise James Cooks (1772-1775) machte (vgl. Rennie 1995). Als zentrales Bildungserlebnis prägte sie seine berufliche wie intellektuelle Existenz.

In der Vorrede zu seinen Kleinen Schriften, in denen er 1788 die wichtigsten seiner verstreut erschienen Aufsätze zusammenfasste, exponiert Forster die wesentlichen Bereiche seiner Forschungen: die „Völker- und Länderkunde“, die „Naturgeschichte“ und die „Philosophie des Lebens“ (AA, V: 345 f.). In der „Völker- und Länderkunde" nimmt er die erfahrene Diversität des Menschengeschlechts in globaler Perspektive in den Blick. Der Mensch wird dabei nicht als Individuum, sondern als Mitglied einer räumlich und zeitlich definierten Kultur untersucht. Dieser Argumentation liegt zugrunde, dass Einheit und Differenz der menschlichen Kultur nur im Rekurs auf die Geographie interpretiert werden können. Thematisiert werden die verschiedenen Kulturformen der Menschheit, die geographischen Räume, spezifischen Ressourcen sowie der gesellschaftlichen Organisationsgrad der unterschiedlichen, während der Reise mit James Cook aufgesuchten Ethnien. Die beständige Wahrnehmung der „Gleichzeitigkeit des Ungleichzeitigen“ (Hans Freyer $)^{2}$ wirft die Frage nach den Ursachen der Entwicklungsunterschiede dieser Völker auf. Schon in seiner Reisebeschreibung (vgl. AA, II, III) deutete Forster die „Völker- und Länderkunde“ als integralen Bestandteil der Wissensform Naturgeschichte und konstituierte eine enge theoretische Verknüpfung zwischen beiden Erkenntnisweisen.

Mit der „Naturgeschichte“ benennt Forster, der in Kassel und Wilna dieses Gebiet lehrte, eine der zentralen, sich strukturell wandelnden Erkenntnisformen des 18. Jahrhunderts. ${ }^{3}$ Die überkommene systematisch-klassifikatorische Perspektive der Naturgeschichte im Sinne Carl von Linnés wird darin Schritt für Schritt durch die genetische Perspektive Buffons ergänzt und partiell auch verdrängt. Forster hat in diesem Verständnis die „eigentliche Naturgeschichte“ einmal als „Geschichte der Entstehung, des Wachsthums, der Veränderungen und Verwandlungen jeder Art" (AA, XIV: 534) ausgelegt. ${ }^{4}$ Die von Buffon angestoßene Dynamisierung der Naturgeschichte wirkte nachhaltig auf angrenzende Wissensgebiete wie die Geschichte, vor allem aber auf die aufklärerischen anthropologi- 
schen Forschungen und Diskussionen (Dougherty 1996). In diesem Kontext wurde das mechanistische Denkmodell ersetzt durch ein organisches. Forster, für den nur ein entwicklungsgeschichtlicher Ansatz dem Untersuchungsgegenstand Mensch gerecht werden konnte, verband in seinen Schriften von Anfang an Naturgeschichte und Anthropologie, um die Natur als zentralen Bestandteil in der Bestimmung des Menschen zu konstituieren.

Seine aufklärerische „Philosophie des Lebens“ repräsentiert die Zurückdrängung des traditionellen, philosophisch-theologisch geprägten anthropologischen Diskurses des homo duplex. Ihr Thema ist die kulturelle und materielle Lebensweltlichkeit des Menschen. In der „Philosophie des Lebens“ nimmt Forster die alltäglichen Denk- und Handlungsweisen in den Blick und entdeckt die Regionalität der Kulturen. Im Rahmen der genetischen Naturgeschichte wird „Leben“ als eine Mannigfaltigkeit von Kräften gedacht, deren Wirkungsweisen nachgewiesen werden sollen. Mit seinem Ansatz der „Philosophie des Lebens“ wendet sich Forster gegen eine Trennung einzelner Erkenntnisinteressen und kann schließlich „Lebensphilosophie“ als Synonym des Begriffes „Kultur“ verwenden, der die „Natur des Menschen“ ebenso wie deren Veränderungen, also die Geschichte des Menschen umfasst. Und Geschichte wiederum setzt er gleich mit der Transformation der „physischen Kultur“ zur ,sittlichen Kultur“. Insofern hat die „Philosophie des Lebens“ ihr Komplement in der „Kulturgeschichte“ (vgl. Garber 1992, 1994, 1995).

Die von Forster genannten Ansätze anthropologischen Forschens korrespondieren mit einem epistemologischen Strukturwandel, in dem das hochaufklärerische rationale Systemdenken Schritt für Schritt durch eine empirische Orientierung ersetzt wurde. Eine sensualistische Erkenntnistheorie wurde als Voraussetzung für eine gemeinsame Betrachtung von Individuum und Gattung behauptet (Kondylis 1986), eine Trennung einzelner Erkenntnisinteressen zugunsten einer integrativen Untersuchung des Menschen abgelehnt. So sucht Forster etwa seine authentischen Erfahrungen mit kritisch eindringender Theorie $\mathrm{zu}$ vereinigen, wenn er formuliert: ,,Mit den lebendigen Eindrücken, welche nur die unmittelbare Anschauung des Objekts, und sonst nichts, geben kann, gieng ich an die Quellen der Länder- und Völkerkunde, schöpfte dort und prüfte zugleich." (AA, V:345) Und die Perspektive der „Philosophie des Lebens“ repräsentiert die Hinwendung zu einer genetischen, biologisch inspirierten Denkform, unterstellt sie doch selbstreferentielle Systeme von Kulturengenese in Analogie zu Naturabläufen. 
Forster hat seine anthropologischen Erkenntnisinteressen nie systematisch konzeptualisiert (vgl. van Hoorn 2004), er war kein Systematiker. ${ }^{5}$ Seine bewusste Vermittlung einer gleichzeitig ethnologisch-kulturanthropologischen und anatomisch-physiologischen Anthropologie repräsentiert eine Variante des spätaufklärerischen „anthropologischen Unitarismus“ (Sergio Moravia). Auch wenn Forster nie ausdrücklich von einem Konzept einer „Wissenschaft vom Menschen" gesprochen hat, schrieb sich sein Erkenntnisinteresse doch in die Debatten über die Wissensform einer „Wissenschaft vom Menschen“, eines Strukturelements der späten Aufklärung, ein (vgl. Moravia 1970, 1982; Chappey 2002; Bödeker/Büttgen/ Espagne 2008). Dieser Diskurs, dessen Gegenstand sich nie als wissenschaftliche Disziplin institutionalisierte, war eine Konstellation unterschiedlicher Disziplinen mit fließenden Grenzen.

Forsters verstreute Ansätze der Konzeptualisierung einer „Wissenschaft vom Menschen“ werden im Folgenden in drei, sich partiell überschneidenden Durchläufen analysiert. ${ }^{6}$ Zunächst gilt es, die zentralen epistemologischen Voraussetzungen von Forsters anthropologischer Argumentation auszuloten. Dazu zählen die Naturalisierung und Historisierung des Menschen und mit ihnen korrespondierend die Historisierung der Natur und die Naturalisierung der Geschichte. Danach werden in zwei Abschnitten einige der Vorstellungen von Forsters Anthropologie, die von ihm so genannte "physische“ Anthropologie und - ihr Komplement - die „sittliche“ Anthropologie in den Blick genommen. Sie können ohne ihre wechselseitigen Bezüge nicht hinreichend verstanden werden. Anschließend werden wissenschaftstheoretische und methodologische Positionen von Forsters eklektischer Erkenntnistheorie analysiert: eine Kombination von Erfahrung und Konstruktion und eine Verbindung von Natur- und Kulturwissenschaften.

\section{„Anthropologische Wende“}

Im Kontext der Neukonstituierung der Wissenschaften um 1750 weitete Forster ebenso wie seine Zeitgenossen den Naturbegriff aus. Die Gesamtheit der Strukturen und Prozesse zusammenfassend kann „Natur" bei Forster zu einem umfassenden Systembegriff aufsteigen. $\mathrm{Zu}$ den weitreichenden Konsequenzen dieser Aufwertung gehört, dass Forster „Natur“ nicht mehr länger der „physischen und sittlichen Bestimmung" (AA, V: 194) des Menschen entgegensetzen konnte. Dazu bedurfte es zweier Voraussetzungen: erstens der Aufhebung der 
Grenzen zwischen dem Körper - als bloßes physiologisches Substrat betrachtet - und dem Seelenleben sowie zweitens des Blicks auf die Gesamtheit der Menschen und der in ihnen wirksamen Verhältnisse von Instinktbindung und Umweltwahrnehmung. Der überkommenen Vorstellung des „homo duplex“ setzte Forster das Konzept der Organisationskräfte entgegen. Darunter sind die Kräfte zu verstehen, die eine Ausdifferenzierung und Weiterentwicklung des Menschen als eines komplex organisierten Lebewesens ermöglichen, und die den Menschen als eine Einheit von Körper und Geist organisieren. Dass sich dieses Konzept im Rahmen eines neuen Begriffs von Kosmos konstituierte, belegt Forsters Antrittsvorlesung in Kassel von 1781, „Ein Blick auf das Ganze der Natur“ (AA, VIII: 77-97).

Die damit implizierte Naturalisierung des Menschen stand im Kontext einer Diskussion von europäischen Dimensionen. Neben Johann Gottfried Herder kann Forster als entschiedener und umsichtiger deutscher Theoretiker dieser Idee gelten. Sie setzt die These einer gleichbleibenden physiologischen Struktur des Menschen voraus. Mit dieser Begründung machte Forster den Menschen zum Forschungsgegenstand der Naturhistorie, um ihn mit deren methodischen Arsenal untersuchen zu können. Gleichzeitig sollten damit nach Forster auch alle jene Ansätze, Hypothesen und Mutmaßungen über die menschliche Natur und das menschliche Geschlecht, die unabhängig von empirischem Datenmaterial entfaltet wurden, zurückgewiesen und ausgeschlossen werden (vgl. van Hoorn 2006). Die These von der Uniformität und Konstanz der menschlichen Natur hat axiomatischen Charakter für Forsters Theoriebildung und ist zugleich auch methodisches Korrektiv. Die unterstellten konstanten Merkmale der menschlichen Natur bezieht Forster durchweg auf die Vielfalt der menschlichen Natur und Kultur. Danach beruht die Diversität der Menschheit auf einem Ursachenbündel physischer Determinationen, die in bestimmter Weise auf die Triebe, Empfindungen oder den Verstand der Menschen einwirken. „[D]i.e Natur des Menschen“ ist für den Weltreisenden Forster ,zwar überall klimatisch verschieden, aber im Ganzen, sowohl der Organisation nach, als in Beziehung auf die Triebe und dem Gang ihrer Entwickelung, specifisch dieselbe" (AA, II: 253).

Da Forster - zugespitzt formuliert - die Einheit von „Mensch“ und „Natur“ behauptete, kann der Mensch bei ihm nicht unabhängig von seiner kulturellen Entwicklung betrachtet werden, kann es keine „Kultur" des Menschen geben, die nicht auch von dessen Verhältnis zur „äußeren Natur“ geprägt ist. Die Ausbildung des „physischen“ Menschen verdankt sich nach Forster dem Gebrauch der Sinne. Dieser ist wiederum selbst die Bedingung der Möglichkeit der Kul- 
turgenese des Menschen. Folgerichtig bildet auch das Verhältnis von Natur und Vernunft nicht mehr länger einen sich ausschließenden Gegensatz: Die Entfaltung der Vernunft in der Geschichte wird als naturhafter Prozess interpretiert. Nicht zuletzt gründete Forsters Entschluss, die Differenz zwischen den Wissensformen Natur und Mensch bewusst niedrig zu halten, in seinem ethnologischen Erkenntnisinteresse.

Im Sinne einer genetisch argumentierenden Naturhistorie begreift Forster den Menschen als ein „unfertiges Wesen“. Gerade in der „Unbestimmtheit“ der Eigenschaften des Menschen sieht er die Voraussetzung für seine Entwicklung. Je weniger determiniert die Anlagen der menschlichen Wesen sind, desto größer ist nach Forster die Amplifikationspotenz ihrer „Vermögen“ (vgl. Nowitzki 2006).

Die „Unbestimmtheit“ menschlicher Grundausstattung denkt Forster auch im Begriff der Perfektibilität, einer geschichtsphilosophischen Grundannahme der europäischen Aufklärung. Darunter versteht er die prinzipielle Vervollkommnungsfähigkeit des Menschen, die er allerdings als relativ begreift, denn ,,vom Menschen bis hinab zum Stäubchen, ist jede Form in der Natur vollkommen in ihrer Art, und sicherlich leidet dieses große Gesetz keine Ausnahme in den verschiedenen Schattierungen des Menschengeschlechts" (AA, V: 330). Damit ist Perfektibilität als ein Prinzip bestimmt, das der Natur inhärent ist. Der Weg der Vervollkommnung wird allerdings von Forster keineswegs als eine kontinuierlich aufsteigende Linie gedacht, und erst recht betrachtet er sie nicht als etwas, das sich ohne aktives Bemühen des Menschen gleichsam wie von selbst vollzieht. Forsters Verteidigung der Perfektibilität richtet sich gegen Rousseaus grundsätzliche Unterscheidung des „Naturmenschen“ vom „Kulturmenschen“. Er erklärt den „Naturmenschen“ für „ein widersinniges Unding“ (ebd.: 262) ${ }^{7}$ und hebt den Menschen gleichsam von vornherein aus dem Naturzusammenhang heraus, gibt es doch für ihn den Gegensatz von Natur und Kultur nicht. Bei Forster ist der Mensch schon immer mit dem „Bewußtsein eines abstrakten Ich“ ausgestattet oder, besser, ,beschenkt" (ebd.: 195).

Der „Unbestimmtheit“ der menschlichen Natur entspricht schließlich die Selbsterhaltung bei Forster (vgl. Mulsow 1995). Das aufklärerische Konzept hebt sich grundsätzlich ab von der traditionellen Lehre der Fremderhaltung des Menschen. Selbsterhaltung bedingt nach Forster "Wachsthum“ und „Bewegung“ (AA, VIII: 209). Er teilte mit vielen seiner Zeitgenossen die Grundüberzeugung, dass der Mensch das prinzipiell entwicklungsoffene 
und nicht von der Natur festgestellte Wesen ist, was wiederum die Voraussetzung dafür ist, dass der Mensch durch und durch geschichtlich ist (vgl. Dreitzel 1981). Der Mensch verändert sich nach Forster in dem Maße, in dem er sich der Außennatur oder einer Fremdkultur assimiliert. Insofern konstruierte Forster die Historisierung des Menschen als Abfolge von Stufen der Vergesellschaftung, die wiederum qualitative Kulturdifferenzen bedeuten. Die Historisierung des Menschen ist dann streng genommen eine genetisch konstruierte Theorie der Differenz von menschheitlichen Entwicklungsstadien im Spannungsfeld von - traditionell formuliert Naturzustand und Zivilisationsgesellschaft.

Die menschliche Entwicklung ist von Forster keineswegs als eine kontinuierlich aufsteigende Linie gedacht. Und er betrachtet die Vervollkommnung des Menschen nicht als etwas, das sich ohne aktives Bemühen und entschlossenes Handeln des Menschen wie von selbst vollzieht. Vielmehr fordert er vom individuellen Menschen, ,unablässig an sich selbst zu arbeiten“ (AA, VIII: 213). Die Vervollkommnung ist wie jede Entwicklung überhaupt das "Ergebnis eines Widerstreits einander entgegen gesetzter Kräfte" (Uhlig 1965: 72). Für Forster gibt es ohne „Contrast weder Größe noch Tugend, noch Vollkommenheit" (AA, VIII: 183). Ohne diesen Antagonismus würde in der Welt die „Ruhe des Todes“ (AA, V: 195) herrschen.

Nicht nur der Mensch wurde während der Aufklärung historisiert, sondern auch die Natur (Toulmin/Goodfield 1985: 157 f.). Die seit Mitte des 18. Jahrhunderts einsetzende Historisierung der Natur hat Forster prägnant auf den Begriff gebracht: „[I]n der ganzen Anlage dieser Welt ist alles auf Beweglichkeit, Veränderlichkeit, nicht auf Dauer und Unzerstörbarkeit eingerichtet." (AA, VIII: 87) Das Reich der Natur - mit ihm die Erde, Pflanzen, Tiere aber auch die Menschen - verändert sich danach fortwährend. Deshalb kritisierte Forster auch die Restriktionen, die die Naturhistorie unter den Epigonen Carl von Linnés erfahren hatte: Sie beschränkten die Naturgeschichte auf die ,äußerlichen Gestalten des Körpers" (ebd.: 78) und klassifizierten sie allein nach äußeren Merkmalen. So konnten sie für Forster bloß ein künstliches, unhistorisches System der Natur nach Art des Tableaus entwerfen. Unter dem Einfluss Buffons forderte er dagegen eine Naturbetrachtung, die neben dem gegenwärtigen Zustand, neben den strukturellen und synchronen Beziehungen zwischen den einzelnen Elementen des gesamten Kosmos auch dessen historische Dimensionen, die diachrone Entwicklung in den Blick nehmen sollte (AA, XIV: 534, 600). Daraus spricht die Auffassung eines permanenten Werdens und 
Vergehens, wobei jedes Wachstum seinerseits wiederum Zerstörung voraussetzt.

Forsters Auffassung, dass die Natur sowohl Geschichte hat als auch Geschichte konstituiert, entspricht das temporalisierte Denkmodell der „Kette der Lebewesen“ (vgl. Wyder 1998) in Form einer kontinuierlichen Folge oder besser Stufenleiter, innerhalb der die einzelnen Lebewesen nach dem Maßstab ihrer „Vollkommenheit“ eingeordnet werden. Ein solch lineares Ordnungsschema erlaubte allein Klassifizierungen, markiert jedoch auch den Ausgangspunkt, an dem eine Darstellung der Geschichte der Natur einsetzen kann.

Forster greift über Buffons Historisierung hinaus und weist bereits auf das 19. Jahrhundert, in dem sich die umfassende Historisierung der Natur vollzog, wenn er den Erwerb neuer Anlagen durch den Gebrauch der Organe und die Vererbung erworbener Anlagen behauptet:

Eine Folge des allgemeinen Wachsthums ist aber die Ausbildung der Organe und Absonderung der Stoffe, welche zur Hervorbringung derselben Form des Daseins in anderen Individuen unentbehrlich ist. Der Mensch wird zu Fortpflanzung fähig, ehe er zu einer bestimmten Länge und Stärke gelangt, ehe er völlig ausgebildet ist, ehe die Knorpel alle geschwunden sind. (AA, VIII: 187)

Diese Dynamisierung der Naturgeschichte trug entscheidend dazu bei, das mechanistische Denkmodell durch das Leitbild des Organismus zu ersetzen.

Für Teile des aufklärerischen Diskurses ist charakteristisch, dass das Paradigma der verzeitlichten Naturgeschichte strukturbildend für die Erkenntnis der Menschheitsgeschichte wurde (vgl. Roger 1989). Buffons Variante von Naturgeschichte eröffnete traditionsbildend die Möglichkeit, Menschheitsgeschichte als Teil der Naturgeschichte zu interpretieren (vgl. bes. Garber 1992, 1994, 1995). In Deutschland vertrat nicht nur Herder diese Naturalisierung der Geschichte breitenwirksam (vgl. Proß 1999), auch Forster konzipierte Menschheitsgeschichte als Naturgeschichte (vgl. Garber 1999). Dass Natur- und Menschheitsgeschichte einer einheitlichen Betrachtungsweise folgen konnten, liegt wiederum darin begründet, dass Forster beide miteinander verschränkte. Natur und Geschichte bilden so einen sachlichen Zusammenhang: Die Kultur geht aus der Natur hervor, ohne deren Grundlage zu verlassen; sie ist veränderte Natur, und die Menschen verändern in diesem Prozess ihre eigene Natur. Forster hat einmal apodiktisch konstatiert:

Doch der Grübeley wird kein Irrthum leichter, als das unterscheiden, wo nichts abzusondern ist; und so erdichtet sie sich einen Widerspruch zwischen Natur und Kultur, der höchstens in einem willkürlichen Gebrauch der Worte 
liegt. Die Fähigkeit zum Denken, mit allen ihren Folgen, ist unserer Natur so wesentlich innewohnend, als der Trieb zur Nahrung und Fortpflanzung, wenn sie gleich nicht jedem Einzelnen nach Möglichkeit entwickelt wird. Was der Gattung zukömmt, entwickelt sich nicht notwendig in jedem Einzelnen. (AA, V: 162)

Als Konsequenz seiner Prämisse der Einheit von Natur und Kultur entwickelte Forster eine Naturgeschichte der Kulturgenese der Menschheit. Die Geschichte stellt er darin dar als Entfaltung heterogener Anlagen, die sich sukzessiv im Übergang von der physischen Anthropologie zur sittlichen Anthropologie, das heißt von der Sinnlichkeit zur Vernunft des Menschen entwickeln.

Wer die Vorzüge des gesitteten Lebens in diesem Zusammenhang ohne Vorurtheil erwägt, wird nicht in Abrede seyn, dass der Mensch in diesem Zustande erst eigentlich der Natur [...] ein Genüge zu leisten anfängt, und wahrer Mensch, das ist, ein denkendes Wesen wird. (AA, V: 161)

Die räumlich unterscheidbaren Kulturen, die der Ethnologe und Kulturgeograph Forster in synchronistischer Methode vergleichend erforschte, werden in der Perspektive seiner Geschichte der Menschheit als eine Stufenfolge der Kulturentwicklung gedacht.

\section{„Physische Anthropologie“}

Im Modell einer verzeitlichten Naturgeschichte entfaltete Forster eine umfassende „unitarische Anthropologie“ (Moravia), wobei er aus theoretischen Gründen die ,physische“ und die „sittliche Anthropologie" nicht grundsätzlich, sondern nur analytisch trennte. Forster hielt deshalb eine Abstraktion des sittlichen vom physischen Menschen für theoretisch unfruchtbar. Seine anthropologischen Reflexionen setzen dann auch konsequent bei der „physischen Anthropologie“ ein (vgl. van Hoorn 2004). Dabei konzentriert er sich auf zwei Themenkomplexe: die Differenz zwischen Mensch und Tier sowie das Verhältnis von Einheit und Vielfalt des Menschengeschlechts. Die Fragen nach der Unterscheidung des Menschen vom Tier - ein zentraler Aspekt der zeitgenössischen anthropologischen Diskussion, der vor allem als die Differenz zwischen Mensch und Affe erörtert wurde (vgl. Corbey/Theunissen 1995) - sowie die nach dem Verhältnis von Einheit und Vielheit des Menschengeschlechts - das zeitgenössisch nicht zuletzt unter dem Titel „Rasse“ debattiert wurde $^{8}$ - hatten sich Forster auf der Weltreise aufgedrängt.

Vor seiner Landung auf der Insel Malekula war Forster fast ausschließlich mit den überwiegend hellhäutigen Polynesiern in Kontakt gekommen und zu der Auffassung gelangt, dass im Süd- 
pazifik die Mitglieder eines einzigen, wenn auch weit verstreuten, klimatisch ausdifferenzierten Stammes leben, dessen gemeinsamer Ursprung aber durch physisch-anthropologische, linguistische und kulturelle Merkmale belegbar ist. Diese Ansicht wurde radikal in Frage gestellt durch die auf Malekula lebenden, damals noch weitgehend unbekannten dunkelhäutigen Melanesier. Sie kamen Forster wegen ihres äußeren Erscheinungsbildes - lange Arme und Beine, tiefschwarze Hautfarbe, Hässlichkeit - für einen Moment „beynahe als ein Affen-Geschlecht" vor (AA, III: 163). Forster sah sich damit mit dem damals hochaktuellen Problem der eindeutigen Grenzziehung zwischen Mensch und Affe konfrontiert. Gleichwohl wollte er dem „Orang-Outang-System“ und „,den seichten Köpfen, die ihm nachbeten" (ebd.: 167) - gemeint war insbesondere Lord Monboddo, für den die Sprache nicht zur vom Tier abgrenzenden Definition des Menschen ausreichte - mit diesem Eingeständnis keinen Beweis liefern. Deshalb schließt er in seiner Beschreibung, dass sich die Bewohner Malekulas im „Stande der Natur“ (ebd.: 181) befinden. ${ }^{9}$ Bereits auf dieser Stufe aber sind sie, so Forster, entgegen der Auffassung Rousseaus, perfektibel und verfügen überdies auch über eine Sprache. Für Forster war es gerade die Sprache, die den Menschen als Mensch erkennbar macht.

Bei seiner Erarbeitung von Kriterien der Differenz zwischen Mensch und Affe zielte Forster darauf ab, den Menschen bereits als ein natürliches Wesen prinzipiell vom Tier zu unterscheiden (vgl. van Hoorn: 2004) und die geistigen, die kulturellen Möglichkeiten und Ausprägungen des Menschen aus dessen Körperlichkeit zu erarbeiten. Dabei spielte für ihn im Gegensatz zu Herder das Konzept des ,aufrechten Ganges“ keine Rolle. Herder, der die überragende Stellung des Menschen in der Tierwelt emphatisch auf die aufrechte Körperhaltung des Menschen zurückführte, bleibt für Forster in anthropomorpher Bildlichkeit stecken:

\footnotetext{
[Herder] lässt mir z. B. die Natur zu sehr auf menschliche Art allegorisieren in ihren Werken. Ich kann mir nicht vorstellen, dass gerade die aufrechte Stellung des Menschen ein Bild seiner Vollkommenheiten und Vorzüge sein soll. Der aufrecht gehende Mensch hat freilich Vorzüge; allein wer bürgt uns, dass es in den Augen der Natur edler und geistiger ist, den Kopf hoch, als niedrig zu tragen? Das nenne ich aus menschlichen Begriffen allegorisiert. [...] Tragen denn nicht alle Vögel den Kopf in die Höhe; am meisten die allerdummsten, die Pinguins? (AA, XIV: 327)
}

Sich auf die Forschungsergebnisse Campers und Soemmerings berufend, nach denen den Affen aus anatomischen Gründen die Fähigkeit fehle, artikulierte Laute hervorzubringen und eine Sprache als Ausdruck von Vernunft und Seele zu entwickeln, ist für Forster - vor dem Hintergrund der Stufenleiter argumentierend - 
die Grenzlinie zwischen Mensch und Tier eindeutig gezogen. Die Bedeutung der durch die Sprachfähigkeit gezogenen Grenze zwischen beiden Gattungen hebt er am 5. Juli 1785 in einem Brief an Soemmering ausdrücklich hervor:

Du könntest allenfalls noch begreiflich machen, dass der Mohr, der dem Weißen so weit nachsteht, hauptsächlich deswegen, weil er die Sprachorgane wie der Weiße hat, folglich sprechen kann, folglich eines Grads von Vernunft fähig ist, unter die Menschen gezählt werden müsse; da es schiene, als hätte die Natur um die Nuance gut zu unterscheiden, gerad den menschenähnlichsten Affen durch die beiden Säckchen die Sprache genommen oder versagt, damit aus ihrer im übrigen doch schon etwas verschobenen Organisation, nicht ein sprechendes Geschöpf mit einer schiefen, falschen Vernunft herauskäme. (AA, XIV: 293)

Da sich für Forster die Sprachfähigkeit des Menschen und seine Denkfähigkeit wechselseitig aufeinander beziehen, gilt für ihn, dass es ,kein Volk ohne Sprache und keine Sprache ohne Vernunft giebt" (AA, V: 195).

Nicht zuletzt die Fähigkeit des Menschen, in jedem Klima zu leben, belegt für Forster die Differenz des Menschen vom Tier (vgl. AA, VI, Bd. 2: 1045 f.). Zur Begründung seiner These beschränkt er sich zunächst ausdrücklich auf die physischen Eigenschaften des Menschen und verweist erst am Schluss seiner Argumentation darauf, dass die Vernunft als Ursache für die Zivilisation mit Kleidung, Behausung, Feuer und Waffen den Menschen erfolgreich gegen die Unbilden des Klimas schützt (ebd.: 1056). Damit spielt er auf die für ihn zentrale wechselseitige Bezogenheit zwischen ,physischer" und "sittlicher" Anthropologie an, wegen der er sich in seiner Argumentation auch durchgehend gegen Rousseaus Auffassung eines tierischen Naturmenschen verwahrt.

Seit der Konfrontation mit der Vielfalt von Ethnien während seiner Weltreise durchzog Forsters Denken die Frage nach der Deutung und Gewichtung dieser Vielfalt. Seine Reflexionen schrieben sich in die seit den 1770er Jahren geführten, disziplinübergreifenden Debatten um die Klassifizierung und - damit unmittelbar verknüpft - die Mono-bzw. Polygenese des Menschengeschlechts ein (van Hoorn 2004: 85 f.): als Ablehnung der geläufigen Modelle der Klassifikation des Menschengeschlechts (vgl. AA, VIII: 193 f.). Die traditionelle theologische Interpretation, nach der das Menschengeschlecht von den drei Söhnen Noahs Sem, Ham und Jafet abstammt, war für den theoretisch reflektierten Naturhistoriker Forster obsolet geworden. Gleiches gilt für die von Linné angestoßene geographische Differenzierung des Menschengeschlechts in die vier "Welttheile“, nach der nicht nach der Fortpflanzung und Abstammung der Menschen gefragt wird, 
sondern die variablen und konstitutiven Merkmale des Menschen thematisiert und vier Gruppen - Americanus, Europaeus, Asiaticus und Afer - konstituiert werden. Die Klassifikation des Menschengeschlechts nach Hautfarbe - hier hatte Forster offensichtlich Kants Bestimmung des Begriffs einer Menschenrasse (Kant 1785) im Blick - erscheint in seiner Schrift erkenntnistheoretisch unzureichend und überdies wenig praktikabel (vgl. Bernasconi 2006). Schließlich spielte er auf Christoph Meiners' „kühnen“ Versuch in dessen Grundriß der Geschichte der Menschheit (1785) an, das Menschengeschlecht in zwei Hauptstämme einzuteilen. Meiners' Klassifikation läuft für Forster darauf hinaus, ,,alle Völker der Erde von einem guten und einem bösen Prinzip abstammen zu lassen" und so die eine Hälfte des Menschengeschlechts als „,geborene Teufel“ zu diffamieren. Meiners' Versuch der Einteilung in zwei ungleichwertige Hauptstämme, der nach Forsters Überzeugung gegen den gesunden Verstand verstößt, fehlt Forster ,nichts als - ein Beweis“ (AA, VIII: 193). ${ }^{10}$

Aus der Kritik an den zeitgenössischen Versuchen, die Vielfalt des Menschengeschlechts zu deuten, zieht er den Schluss: „So thue dann auch ich ohne Scheu das Bekenntnis, dass ich anderwärts mich Raths erholen muss, um die Abstände zwischen verschiedenen Nüancen im Menschengeschlecht zu messen." (Ebd.) Forster legte bewusst keinen eigenen Versuch der Einteilung vor; vielmehr konzipierte er, um das theoretische Problem zu bewältigen, einen dynamischen Entwurf von Entwicklungsstufen des Menschengeschlechts.

Den beiden damals geläufigen Denkmodellen zur Vielfalt der Menschheit - der Klimatheorie (Fink 1987; van Hoorn 2003) und der Theorie einer einheitlichen Abstammung - stand Forster kritisch distanziert gegenüber. Schon während seiner Weltreise sah er sich Schritt für Schritt gezwungen, sich von der vorherrschenden Doktrin zu lösen, nach der das Klima als einzige Erklärung für das unterschiedliche Aussehen der Menschen, für ihre unterschiedlichen Verhaltensweisen und für ihre asymmetrischen kulturellen Entwicklungen zu gelten habe. Wenn die ",affenähnlichen“ Bewohner Malekulas Menschen waren - wie Forster ausdrücklich konstatierte - , dann musste seiner Meinung nach die Klimatheorie als Erklärungsmodell für die Vielfalt des Menschengeschlechts grundsätzlich infrage gestellt werden. Seine zunehmende Skepsis hält Forster 1774 fest: „Ich meines Theils gestehe dem Clima bey weitem keinen so allgemeinen und allwirksamen Einfluss zu." (AA, III: 179) Im weiteren Verlauf seiner Reise verlor die Klimatheorie für Forster immer weiter an Plausibilität, so etwa auf Neu-Kaledonien, 
wo er das Verhalten und die Physiognomie der Einwohner im Widerspruch zur Klimatheorie sah:

Je sparsamer nun allhier die Natur ihre Güther ausgetheilt hat, desto mehr ist zu bewundern, daß die Einwohner minder wild, mißtrauisch und kriegerisch, als auf Tanna, und vielmehr so friedlich und gutartig waren! Eben so merkwürdig ists, daß sie, bey aller Dürre des Landes, und bey ihrer kärglichen Versorgung mit Pflanzenspeisen, dennoch von größerer und muskulöser Leibesstatur sind, als die Tanneser. (Ebd.: 326)

Seine Reiseerfahrungen zwangen Forster, das Verhältnis zwischen Mensch und Umwelt als weitaus komplexer anzusehen, als dies die zeitgenössischen Klimatheorien vermochten. ${ }^{11}$

Die Unterschiede zwischen den beiden im pazifischen Raum ansässigen Rassen der Polynesier und Melanesier schienen Forster in einer ursprünglichen Verschiedenheit der autochthonen Urbevölkerung zu gründen, die jedem dieser Stämme ,,jeweils seine eigene unzerstörbare und gleichsam eingeprägte Gestalt kenntlich an charakteristischen Zeichen" vererbt hatte (AA, VI, Bd. 1: 93-137, bes. 96 f., 101 f.). Explizit exponierte er die These der zwei „Hauptabtheilungen“ der Menschen in der Südsee in seinem 1786 erschienenen Aufsatz „Noch etwas über die Menschenrassen“ (AA, VIII: 130-156). Die offenbaren Differenzen hatten ihn auf die theoretische Möglichkeit einer polyphyletischen Herkunft des Menschengeschlechts gebracht. Im Anschluss an die anatomischen Forschungen Soemmerings, der zu dem Schluss gekommen war, der Afrikaner stehe mit seiner physischen Konstitution „dem Affen näher als dem Europäer" (Soemmering 1785: XX), erörtert auch Forster die wesentlichen physischen Differenzen im Menschengeschlecht. Freilich betonte Soemmering, die „Neger“ seien nichtsdestoweniger „wahre Menschen, so gut wie wir“, und hielt ausdrücklich fest, sie seien ,nach höchstwahrscheinlichen Gründen, die uns Naturgeschichte, Philosophie und schriftliche Nachrichten darbieten, von einem gemeinschaftlichen Stammvater mit allen Menschen entsprossen" (ebd.: XXII). Forster waren diese Unterschiede zwischen den menschlichen Rassen wichtig genug, um ihre getrennte Abstammung für möglich zu halten. Er nutzte die klimaunabhängigen physischen Differenzen im Menschengeschlecht theoretisch konsequenter und auch mutiger als Soemmering. In Übereinstimmung mit dem zeitgenössischen essentialistischen Artbegriff und gegen Kants hypothetisch formulierte und entwicklungsgeschichtlich argumentierende Deutung des Terminus Rasse formulierte Forster die These eines möglichen polygenetischen Ursprungs des Menschengeschlechts. Für ihn lässt sich sowohl aus naturhistorischer als auch aus moralphilosophischer Sicht die 
Polygenese ebenso plausibel begründen wie die Monogenese (vgl. Stumman-Bower 2004).

Allerdings sind Forsters Reflexionen über die Frage eines monogenetischen oder polygenetischen Ursprungs des Menschengeschlechts wenig kohärent. Da er die Frage auf dem zeitgenössischen Stand der Forschung für nicht lösbar hielt, lenkte er das Augenmerk darauf, „,dass alles in der Schöpfung durch Nuancen zusammenhängt" (ebd.: 187). Und orientiert an der Anerkennung der Alterität warf er folgende politisch brisante Frage auf:

[...] indem wir die Neger als einen ursprünglich verschiedenen Stamm von weißen Menschen trennen, zerschneiden wir da nicht den letzten Faden, durch welchen dieses gemisshandelte Volk mit uns zusammenhieng, und vor europäischer Grausamkeit noch einigen Schutz und einige Gnade fand? Lassen Sie mich lieber fragen, ob der Gedanke, dass Schwarze unsere Brüder sind, schon irgendwo ein einziges Mal die aufgehobene Peitsche des Sklaventreibers sinken ließ? (AA, VIII: 154)

\section{„Sittliche Anthropologie"}

Forsters Ligatur der physischen und der sittlichen Anthropologie findet ihr Komplement in seiner Konzeption einer Kulturgeschichte der Menschheit, die ihr Paradigma in der verzeitlichten Naturgeschichte hat. In seiner Menschheitsgeschichte fragt Forster danach ,wie der Mensch 'naturgemäß' zu dem geworden ist, was ihn als Kulturwesen ausmacht" (Garber: 1999: 32). Naturgeschichte und Menschheitsgeschichte kann er deshalb aufeinander beziehen, weil für ihn einerseits die Transformation der physischen Natur in die sittliche Kultur mit Geschichte identisch ist. Andererseits und zugleich bilden Natur und Geschichte in seiner Sicht einen metaphorischen Zusammenhang, denn Forster denkt die Menschheitsgeschichte im Modell des natürlichen Wachstums eines Individuums (vgl. Rohbeck 2006).

Den Zusammenhang von Mensch und Natur betrachtete Forster durchaus als ein Herrschaftsverhältnis: Es ist der Mensch, der die Ordnung und Schönheit in der Natur konstituiert. Seine Eingriffe in die Natur erschaffen eine „zweite Natur“, die Kultur, in der die Unordnung der ersten Natur aufgehoben wird. So hielt Forster einmal apodiktisch fest:

Wie schön ist sie nicht, diese gebaute Natur! Wie hat die Sorgfalt des Menschen sie so glänzend und prächtig geschmückt! Er selbst, der Mensch, gereicht ihr zur vornehmsten Zierde; er ist das edelste Erdengeschöpf; er pflanzt ihre kostbarsten Keime fort; indem er sich selbst vermehrt. Auch sie, die Erde scheint mit ihm sich zu vermehren. Alles, was sie in ihrem Schoße verbarg, bringt er durch seine Kunst ans Licht. (AA, VIII: 95 f.) 
Forster analysiert in seiner Geschichte der Menschheit nicht nur die synchrone Vielfalt der menschlichen Natur und Kultur, sondern erweitert diese räumliche Perspektive durch seine diachrone Fragestellung. Untersuchungsgegenstand der Menschheitsgeschichte als Kulturgeschichte sind die zeitgenössischen sowie die historischen Ausprägungen der vergesellschafteten Menschen. In Forsters Argumentation wird die mechanistische Metapher von Buffons „Weltmaschine“ beziehungsweise der "Mechanismus des Ganzen“ (vgl. AA, VIII: 92 f.), der den für ihn so wichtigen Zusammenhang aller Teile der Natur konstituiert, allmählich abgelöst durch den Begriff des Organismus (vgl. ebd.). Dessen Entwicklungsdynamik konzipierte Forster in Analogie zu der ,vis essentialis“ von Caspar Friedrich Wolff und dem „Bildungstrieb“ "Johann Friedrich Blumenbachs (vgl. ebd.). ${ }^{12}$ Ein weiteres Indiz dafür, dass Forsters Modell einer von dynamischen Kräften angetriebenen Menschheitsgeschichte im Kontext der Naturgeschichte entstand.

Für Forster erfüllte sich die Natur des Menschen in seiner Geschichte wie sich umgekehrt seine Natur aus seiner Geschichte erschließen lässt. Er synchronisierte gleichsam die historische Entwicklung des sinnlichen und geistigen Menschen. Die Aufhebung des Gegensatzes von Natur und Kultur schließt die seit der Antike überkommene Polarisierung einer naturzuständlichen Urgeschichte des Menschen und einer entfalteten zivilisierten Lebenswelt aus. Konsequent lehnte Forster deshalb die Annahme eines Naturzustandes ab. Es konnte für ihn keinen ,bloß thierischen Stand der Natur" (AA,VI: 150) geben. Diese dezidierte Abgrenzung markiert zugleich den Ausgangspunkt historischen Denkens im Rahmen der Naturgeschichte. Forster teilte es mit den Vertretern der schottischen Moralphilosophie, es lässt sich als Historisierung des Naturzustandes interpretieren (vgl. Medick 1973).

Mit der Abkehr von der dualistischen Kontrastierung von „Wildheit" und „Zivilisation“ korrespondiert bei Forster die Aufhebung der These vom „edlen Wilden“ (vgl. Kohl 1981). Er konnte die Südsee-Insulaner während seiner Reise um die Welt nicht mehr länger als glorifizierte ,edle Wilde“ interpretieren, sondern sie nur als Ethnien mit jeweils eigentümlicher Kultur analysieren. Die scheinbar „primitiven“ Kulturen legte er so als Gesellschaftsformen aus, die in historischer Perspektive der eigenen europäischen Vergangenheit durchaus entsprechen könnten.

Wie die Ablehnung der Lehre vom „edlen Wilden“ ist auch die Polemik gegen den Naturzustand bei Forster weitgehend in die Form einer Kritik an Rousseau gekleidet. So schrieb er am 24. April 1784 an seinen Freund Soemmering, dass er keineswegs „,mit 
dem mürrischen Genfer [...] ein Waldmensch werden und der Vernunft entsagen“ möchte; er zweifele am Glück, „welches der inconsequente Theorist dem Wilden andichtete, ohne selbst davon kosten zu mögen“ (AA, V: 363) Das hängt systematisch auch mit einer bei Forster erkennbaren, freilich nur rudimentär ausgebildeten normativen Theorie der zivilisatorischen Entwicklung zusammen.

Im Rahmen seiner naturhistorisch begründeten „Philosophie des Organischen“ analogisiert Forster die Geschichte des Individuums mit der Geschichte der Menschheit. Forster, der aus tatsächlichen, beobachtbaren Entwicklungen des einzelnen Menschen auf die Entwicklung der Gattung schließen wollte, stand damit im Streit zwischen Kant und Herder eindeutig auf der Seite Herders. ${ }^{13}$ Und seine Entwicklung des Verhältnisses von Individuum und Gattung ist stärker den schottischen Debatten geschuldet denn der aufklärerischen geschichtsphilosophischen Konzeption (vgl. Meyer 2008; Uhlig 2003). Forster dachte die Geschichte der Menschheit im Modell der natürlichen Entwicklung des Individuums und ordnete die aufeinander folgenden Etappen der Entwicklung des Einzelnen, die biologisch vorgegeben sind, der kulturellen Entwicklung der Menschheit zu. Die Schilderungen der vierphasigen Entwicklung des einzelnen Menschen fungierten bei ihm gleichsam als Vorlauf zur Beschreibung der verschiedenen historischen Kulturstufen der Menschheit, denn ,ganze Völker scheinen jene verschiedenen Stufen der Bildung hinanzusteigen, die dem einzelnen Menschen vorgezeichnet sind“ (AA, VII: 190). Allerdings reicht Forsters Argumentation über eine bloße Analogie zur individuellen Entwicklungsgeschichte insofern hinaus, als er durch die Perfektibilität ,der Tätigkeit nach Außen und der Denkkraft" (Garber 1999: 36) ein Kultursystem entstehen sieht. ${ }^{14}$

Im Paradigma einer verzeitlichten Naturhistorie entfaltete Forster das Modell einer Stufenleiter der kulturellen Entwicklung, eine diachrone Theorie der menschlichen Geschichte, in der alle bisher ausgebildeten Formen der materiellen und ideellen Kultur aufeinander bezogen sind. Mit diesem Entwurf, der einer „metaphysischen Einteilung" (AA, VIII: 190 f.) der Menschheitsgeschichte entgegenwirken sollte, griff Forster auf die traditionelle Lebensaltermetaphorik für die Abfolge der Geschichtsperioden zurück. Den vier Phasen der Individualentwicklung „Wachstum, Pubertät, Stillstand“ und „Hirnhärtung“ ordnet er jeweils eine „Hauptbestimmung“ zu, nämlich „Selbsterhaltung, Fortpflanzung, Wirksamkeit nach Außen und Rückwirken in sich selbst" (AA, VIII, 189), und überträgt sie auf die ökonomischen, sozialen, politischen und kulturellen Strukturen der menschlichen Vergesellschaftung in historischer Perspektive (vgl. Meyer: 2008). 
Auf der ersten Stufe sind die Menschen demnach gezwungen, sich ausschließlich der Selbsterhaltung zu widmen; die vorrangig mit der Nahrungssuche zur Erhaltung des eigenen Lebens beschäftigten Menschen leben in unorganisierten „Haufen“ ohne gesellschaftliche oder politische Strukturen. Auf dieser Stufe bloßer Selbsterhaltung stehen für Forster die zeitgenössischen „Wilden“. Die zweite Entwicklungsstufe ist nach Forster durch eine grundsätzliche Verbesserung der materiellen Lebensgrundlagen der Menschen gekennzeichnet, so dass sich die "Haufen“ nicht nur vermehren, sondern auch organisieren können. Mit diesem „Zeitraum der Vermehrung“, der der durch den Geschlechtstrieb bestimmten Stufe der Pubertät entspricht, korrespondiert für Forster die Entstehung von Knechtschaft und Despotismus. China, Indien und Afrika gelten ihm als repräsentativ für die Entwicklungsstufe. Der Kampf um gesellschaftliche Macht und um Reichtum macht dann die dritte Entwicklungsstufe der Menschheit aus. Die „Barbaren“ der griechischen und römischen Antike dienen Forster als Beispiele für diese Stufe, die durch ,,gewaltsames Streben nach Herrschaft und Genuss" geprägt ist. Erst auf der vierten Stufe besteigt laut Forster die „Vernunft [...] ihren Thron.“ (AA, VIII: 188). Diese Stufe der verfeinerten Empfindung und des Verstandes wurde nach Forster erst dreimal erreicht, und zwar sowohl im Athen und im Rom der Antike als auch im zeitgenössischen aufklärerischen Europa.

Seine vier Kulturstufen definierte Forster auch als „muskularische, spermatische, heroische und sensitive Kultur" (AA,VII: 191 f.). $\mathrm{Da}$ er den einzelnen Entwicklungsstufen gesellschaftlich-politische Strukturen unterlegt, verschränkt er die organische Perspektive mit der "four stages theory“ der schottischen Schule. Es liegt nahe, dass Forster in Anlehnung an Adam Ferguson die vier Stufen als Jagd-, Viehzucht-, Ackerbau- und Eigentümergesellschaft auslegte (vgl. Meyer: 2008). Damit konstituierte er Verbindungslinien zwischen anthropomorphen und sozialen Klassifikationen der Menschheitsentwicklung, welche sich gleichsam im Organismus der Menschheit selbst vollzieht:

Nur solche Völker, die in ihrer früheren Periode der Wollust glücklich entgangen, und in den Armen der Freiheit zur männlichen Stärke herangewachsen sind; können und müssen zuletzt den höchsten Gipfel der Bildung ersteigen, wo die ganze Energie unseres Wesens sich in feineren Werkzeugen der Empfindung und des Verstandes am thätigsten erweiset. (AA, VIII: 189)

Allerdings bleibt undeutlich, wie Forster die anthropomorphen und die soziopolitischen Perspektiven verknüpft. 
Seine lineare, jedoch nicht teleologische Abfolge von Entwicklungsstufen ist so angelegt, dass am Ausgang die Entfaltung aller Anlagen des Menschen erkennbar wird. Allerdings unterscheidet sich Forster von seinen Zeitgenossen dadurch, dass für ihn jede der Entwicklungsstufen ein bestimmtes anthropologisches Spezifikum vollständig entfaltet, welches dann im weiteren Geschichtsverlauf seine zentrale Stellung verliert (vgl. AA, VII: 15-28). Am Beginn der menschlichen Geschichte dominiert demnach, zugespitzt formuliert, die physische Anthropologie, während am Ende die reflexive Kultur vorherrscht (vgl. ebd.). In der Perspektive von Forsters Geschichte der Menschheit scheint sich der kulturelle Fortschritt in der Gegenwart auf Kosten des ,,sinnlichen Menschen“ zu beschleunigen, der von der reflexiven Übermacht der Aufklärung in seinen ,physischen“ Möglichkeiten mehr und mehr eingeschränkt wird.

Gerade um sowohl die während der Weltreise erfahrenen verschiedenen Entwicklungsformen und -geschwindigkeiten theoretisch bewältigen zu können, konzipierte Forster ein flexibles Stufenmodell. Die Fähigkeit, einen diskontinuierlichen, ungleichzeitigen und ungleichförmigen Fortschritt, aber auch Stagnationen, Verlangsamungen und Regressionen sowie spezifische regionale kulturelle Ausprägungen erklären zu können, findet sich bereits in Forsters Beschreibung seiner Weltreise. In den 1780er Jahren bildete er dieses komplexe Fortschrittskonzept, in dem sich lineare und zyklische Momente durchdringen, weiter aus: „das Jahrhundert, wie das Menschengeschlecht überhaupt, rückt nicht vorwärts in einem regelmäßigen Schritt, sondern in einer unaufhörlichen Rotation." (AA, XV: 231) Das Ziel der Entwicklung lässt sich nicht bestimmen: „Der Gränzpunkt der fortschreitenden Aufklärung liegt außer unserm Gesichtskreise“ (AA, V: 199).

Forster wollte jedoch nicht nur die Entwicklungsstationen des Menschengeschlechts skizzieren, er stellte sich zugleich auch die Frage nach den Prinzipien dieser Entwicklungsprozesse. Mit Nachdruck vertrat er gegen die Theorie des „Gleichgewichts der Kräfte“ die These, dass eine einseitige Ausbildung der Teile die Bedingung der Möglichkeit der Vervollkommnung des Ganzen ist. Für ihn konstituiert Differenz und Widerstreit den historischen Prozess. Zivilisatorischer Fortschritt kann für Forster dann nur durch Exzentrizität entstehen, das heißt durch die Entfaltung von Teilkräften des Menschen: „Exzentrizität ist [...] eine Bedingung, ohne welche sich der höchste Punkt der Ausbildung [...] nicht erreichen lässt." (AA, IX: 167) Die Menschen können nach Forster allerdings immer nur partielle Vollkommenheit erreichen, was wiederum bestimmte Eigenschaften ausschließt, so dass sich die Gesamtheit des menschlichen 
Anlagepotentials niemals verwirklichen kann (vgl. ebd.: 306-320). Zugleich ist das Exzentrische des Individuellen die Bedingung für die Dynamisierung des Allgemeinen. „Exzentrizität“ wird von Forster bezogen auf den „Antagonismus“. Seine menschheitsgeschichtliche Dimension beschrieb er wie folgt:

Der Wechsel der Verhältnisse, der Zusammenstoß streitender Kräfte, der Contrast entgegengesetzter Ereignisse - diese hin und her strömende Fluth im Ocean der Menschheit läutert und bestimmt überall die Begriffe, und giebt ihnen auch Einfluss auf Handlungen, Tugend und Laster sind daher überall gleichzeitige Erscheinungen; denn auch die Tugend wird durch das Widerstreben möglich; wo weder Feind noch Gefahr vorhanden ist, da giebt es weder Kampf noch Sieg. (AA, V: 196 6f.)

Forsters Interpretation der historischen Prozesse als Folgen eines chaotischen Gegeneinanders menschlicher Interessen rekurriert auf Positionen des antiken Atomismus wie der schottischen Aufklärung. Seine Auffassung, dass Eigensucht und Egoismus eine Dynamik der Geschichte konstituieren, in der sich die negative Antriebskräfte einerseits aufheben und es andererseits ermöglichen, dass das Neue sich gegenüber dem Alten durchsetzen kann, brachte er prägnant auf den Begriff: „Luxus, Kunst und Wissenschaft, die Kinder Einer Geburt, vermählen sich miteinander und bringen eine neue Brut - Ungeheuer und Genien - zur Welt." (AA, IX: 326) Forster machte aber nicht nur Leidenschaften, Krieg und Gewalt, sondern auch Handel und „Industrie" als Antriebskräfte der Geschichte, als Ursachen des Fortschritts aus (vgl. van Hoorn 2004: 229 f.). Die Kumulation von materiellen Reichtümern erklärte er sogar zur Voraussetzung des Umschlags der physischen Kultur in die sittliche (AA, VIII: 47).

Sein Realitätssinn ließ Forster nüchtern einen „Kreislauf der Begebenheiten“, einen „unaufhörlichen Wechsel“ im geschichtlichen Prozess konstatieren (AA, V: 197). Gleichwohl finden sich bei ihm Vermutungen über „das gemeinschaftliche Fortrücken unserer ganzen Gattung nach einem bestimmten Ziele der Vollkommenheit" (ebd.: 292). Solche Prognosen werden aber durchgehend infrage gestellt, denkt Forster doch den Widerstreit der Kräfte nicht nur als ein Mittel zum Zweck, sondern als die Bedingung der Möglichkeit des Lebens überhaupt, als „Puls der Natur“:

In einer Welt, wo die größte Mannichfaltigkeit der gestalten nur durch das vermögen einander zu verdrängen, bewirkt wird, hieße es in der That die einzige bedingung ihres Daseyns aufheben, wenn man diesen immerwährenden Krieg und diese anscheinende Unordnung abgestellt wissen wollte. (AA, $\mathrm{V}: 193)$

So mündet Forsters Argumentation in der Einsicht, dass die Menschen ihre Geschichte nicht zu planen und nicht zu machen vermögen. Seine Konzeption unterscheidet sich von den traditio- 
nellen Menschheitsgeschichten insofern, dass bei ihm die letzte Stufe der Menschheitsgeschichte, die universalistische Kulturbildung im Zeitalter der Aufklärung als Ergebnis eines Kontingenzprozesses, keineswegs einer linearen Verlaufsform folgt. Auch seine Geschichte der Menschheit ist nicht die Geschichte der Spezies Mensch. Wie seine Zeitgenossen ging Forster noch von dem naturhistorischen Axiom der Konstanz der Arten aus. Die Forster'sche Geschichte der Menschheit ist eine Geschichte der Entfaltung der im Menschen angelegten Möglichkeiten zivilisatorischer und kultureller Entwicklungen, deren „Ganzes“ grundsätzlich jedoch erkennbar ist.

\section{"Aufklärerische Erfahrungswissenschaft"}

Im Zentrum von Forsters Erörterungen der methodologischen Grundlagen seines Forschens steht die Diskussion über Spekulation und Empirie. Sie bestimmte bereits die Darstellung seiner Weltreise. Einerseits kritisiert er damit den Wissenschaftler, der nur „nach Factis“ jagt und als Ergebnis lediglich ,einen vermischten Haufen loser einzelner Glieder" vorweisen kann, aus welchen „sich durch keine Kunst ein Ganzes hervorbringen“ (AA, III: 13) lässt. Andererseits lehnt er den bloßen Theoretiker ab, der ohne „hinreichende Kenntnis [...] gewisse Sätze für wahr“ hält und sich ,auf diese Art der Systeme [aufbaut], die von fern ins Auge fallen, aber, bey näherer Untersuchung, uns wie ein Traum mit falschen Erscheinungen betrügen" (AA, VIII: 132). Die naturhistorische Taxonomie Linnés, der die Erscheinungen der Wirklichkeit vereinfachte und auf der Grundlage dieser Reduktion auf Begriffe brachte, markiert für Forster die Grenze einer durch festgelegte Begriffe geprägten Erfassung von Realität (ebd.: 132 f.). Die ,spekulative Philosophie“ Kants ist für Forster „diesem allgemeinen Schicksal [ebenfalls, H. E. B] unterworfen“ (ebd.: 132). Der Rückschluss von begrifflich gewonnener Systematik auf die zureichende Erfassung der Realität kann nach Forster nicht funktionieren:

Mit einem Worte, die Ordnung der Natur folgt unseren Eintheilungen nicht, und sobald man ihr dieselbe aufdringen will, verfällt man in Ungereimtheiten. Ein jedes System soll Leitfaden für das Gedächtniß seyn, indem es Abschnitte angiebt, welche die Natur zu machen scheint; da man aber alle gleichnamige Abschnitte, wie Geschlecht, Gattung, Varietät, überall in gleichen Entfernungen von einander stehen, kann und darf man nicht behaupten. (AA, XIII: 146)

Forster äußerte also grundsätzliche Skepsis gegenüber einer deduktiven Begriffsbestimmung, die sich für ihn immer in Gefahr begibt, an 
empirischen Phänomenen vorbei zu abstrakten, mit der Wirklichkeit nicht übereinstimmenden Aussagen zu kommen:

Wenn also der Satz: dass man in der Erfahrung nur als denn finde was man bedarf, wenn man vorher weiß, wornach man suchen soll [.....] auch seine unangefochtene Richtigkeit hätte: so wäre gleichwohl bey der Anwendung desselben eine gewisse Vorsicht nöthig, um die gewöhnlichste aller Illusionen $\mathrm{zu}$ vermeiden, diese nämlich, dass man bey dem bestimmten Suchen nach dem was man bedarf, dasselbe oft auch zu finden glaubt, wo es wirklich nicht ist. (AA, VIII: 132)

$\mathrm{Zu}$ den Voraussetzungen einer reflektierten Naturgeschichte zählte Forster neben der Unparteilichkeit und der Vorurteilsfreiheit (vgl. AA, V: 285) die Abstinenz von spekulativ gewonnenen „,vorherbestimmten Begriffen“ beziehungsweise „kunstmäßigen Hypothesen“ (ebd.: 185, 397). Gerade diese Forderung verteidigte er nachdrücklich in seiner Auseinandersetzung mit Kant über die Klassifizierung des Menschengeschlechts (van Hoorn 2004: 124 f.). Forster plädiert für eine sich selbst reflektierende und dadurch geschärfte Wahrnehmungs- und Urteilskraft. Prinzipiell zieht er die „offenen Augen“ des „zuverlässigen Empyrikers“ den ,geschminkten [Beobachtungen, H.-E. B.] eines partheyischen Systematikers" vor (AA, VII: 133), weil diese "manches willkürlich in einem vorhinein bestimmtem Lichte“ (ebd.: 134) erscheinen lassen und damit Trugbilder der Wirklichkeit produzieren. Trotz der Betonung eines emphatischen Erfahrungsbegriffs entschied sich Forster jedoch nicht, wie von der Forschung fast durchgehend unterstellt wird, für die induktive, empirische Methode. Er gestand selbst, dass „diese Gegensätze ...vielleicht zu schneidend nebeneinander" stehen und sowohl der „empyrische als der systematische Kopf [...] unter gewissen Umständen die besten Beobachtungen liefern" kann (ebd.: 133). Somit steht seine Wissenschaftsauffassung bewusst in der Mitte zwischen den zwei von ihm verworfenen Extremen der reinen Tatsachenbeschreibung und der Systembildung. Sowohl die Theoriebildung der Hochaufklärung einschließlich kantischer Systemformen als auch die sich herausbildenden und sich im frühen 19. Jahrhundert etablierenden Erfahrungswissenschaften in ihrem positivistischen Selbstverständnis lehnte er ab. Sein besonderes Interesse galt dagegen zeitgenössischen Vorschlägen zur Synthese deduktiver und induktiver Ansätze. Im Sinne von Adam Fergusons Grundsätzen der Moralphilosophie (Ferguson 1772; Oz-Salzberger 1995) beschrieb er 1781 in seinem Vortrag „Blick in das Ganze der Natur" die Methode des Naturforschers ,als eine Pendelbewegung von den empirischen Einzelerscheinungen zu den Einsichten in den Gesamtzusammenhang der Natur, um daraus Gesetzmäßigkeiten ableiten zu können“ (Garber 1999: 36). 
Forster war bestrebt, die losen Glieder empirischen Wissens $\mathrm{zu}$ einem umfassenden Ganzen zusammenzufügen, oder, wie er Heyne gegenüber äußerte, ,,auch naturhistorisch auf den Zusammenhang des Ganzen" (AA, XVIII: 132) zurückzuführen. Sein Vorgehen kann nicht bloß nach der üblichen induktiven Idee des Fortschreitens betrachtet werden. Vielmehr wird die - vor allem an Buffon orientierte - These vom Ganzen der Natur zum heuristischen Ausgangsprinzip der Untersuchungen erhoben und in ein konkretes Forschungsprogramm umgesetzt.

Diese Argumentation setzt die verzeitlichte spätaufklärerische Naturgeschichte voraus, nach der jedes Einzelelement Baustein einer Systembildung sein kann. In der Naturgeschichte folgen Teil und Ganzes einem gemeinsamen Strukturprinzip. Durch die Einführung einer Kraft- und Vitalismustheorie kann mit der Naturgeschichte die Selbstorganisation von Teil und Ganzem als ein organisierter Prozess ausgelegt werden.

In seinen späten epistemologischen Reflexionen ergänzte Forster die „Erfahrung“ und die „Urteilskraft“ durch die „Einbildungskraft" als drittes erkenntnisbildendes Vermögen. Er stellt sie neben das „Beurteilungsvermögen“ und beschrieb sie als das, was „die Verhältnisse der Dinge schnell und richtig auffasst und bemerkt" (AA, V: 299). Dieses dritte Vermögen vermittelt danach offensichtlich zwischen der bloßen Wahrnehmung einzelner Gegenstände und der verstandesmäßigen Beurteilung ihrer Zusammenhänge. Forsters spezifisches Konzept der Einbildungskraft ähnelt den Bestimmungen, die Kant der Einbildungskraft als einem synthetisierenden Vermögen in der Kritik der reinen Vernunft gibt (vgl. Kant 1957 [1790]: 237622). Den Ursprung dieses Konzeptes sah Forster 1792 im engen Zusammenhang mit seiner Ausbildung und seiner Reise mit James Cook, wie er in seinen Vorlesungen über allgemeine Naturkenntnis festhielt:

Ich verdanke dieser Schiffahrt die Entwicklung einer Anlage, welche von Kindheit an meine Richtung bestimmte, nämlich eines Bemühens, meine Begriffe zu einer gewissen Allgemeinheit zurückzuführen, sie zur Einheit zusammenzubinden und dadurch der Ahndung des Ganzen mehr Leben und konsequente Wirklichkeit in mir selbst verschaffen (Forster [1792] zit. nach Jahn 1994: 171)

Auf der Konstruktionsleistung des Forschers insistierend ebnete Forster den Gegensatz von Subjekt und Objekt weitgehend ein. Da für ihn der Beobachter Teil des Erkenntnisprozesses ist, verändern sich im Akt der Erkenntnis Subjekt und Objekt. Dieses Verfahren eines reflexiven Erkennens hat Forster bereits in der Vorrede zur „Reise um die Welt" postuliert (AA, II: 7-17). Für seine wissenschaftliche Praxis 
nutzte Forster den Begriff der ,unphilosophische[n] Art zu philosophieren" (AA, XV: 233), konstituiert durch die methodischen Operationen der Beobachtung, des Vergleichs und der Analogie.

Forster forderte einen ,eigenen Beobachtungsgeist" (AA, V: 242, vgl. Bödeker 2006) des Naturhistorikers ein, der konzeptuell an der Tradition der naturhistorischen Deskriptionsmethode Buffons orientiert ist. Die ,umsichtige Beobachtung des menschlichen Lebens" (AA, II: 297) ist für Forster ein erster Schritt zur Erkenntnis nicht nur fremder Kulturen. Sie ist allerdings kein spontaner Wahrnehmungsakt, der voraussetzungslos erfolgt. Forster betonte ausdrücklich, dass mit einem "Schema im Kopfe“ (AA, V: 393), das sich aus „Vorkenntnissen“ konstituiert, beobachtet wird. Deshalb forderte er den sich selbst beobachtenden Beobachter, davon ausgehend, dass nur ein solcher weiß, aus welcher Perspektive er einen Gegenstand fixiert, welche eigenen Vorurteile in die Beobachtung eingeflossen sind. Daher kann nur er allein kontrollierbare Aussagen über seine Tatsachenerkenntnis machen. Vorurteile beim Beobachten können nicht vermieden werden, so Forster, weil sie gleichsam die kulturelle Vorgabe jeder Erfahrung sind, da jeder Erkenntnisakt und jedes Erkenntnisobjekt kultur- und geschichtsvermittelt sind. Und er forderte vom Beobachter, diese Perspektive nicht nur zu reflektieren, sondern dies in der späteren Deskription seiner Beobachtungen auch darzulegen. Der Leser muss wissen, so konstatierte Forster in der Einleitung zu seiner Weltreise, ,wie das Glas gefärbt ist, durch welches ich gesehen habe" (AA, II: 13). Methodisch konsequent reflektierte er darauf hin nicht nur seine subjektiven Perspektiven auf die beobachteten Gegenstände, Kulturformen und Menschen, sondern problematisierte auch seinen teilweise empfindsamen, subjektiven Schreibstil (vgl. ebd.).

Diese Einsicht hinsichtlich von Wahrnehmungsverzerrungen hat Forster nicht nur programmatisch formuliert; in seinen Veröffentlichungen war er bestrebt, sie auch umzusetzen. Freilich sind auch sie nicht frei von verzerrenden Perspektiven; die Grenzen Forsters Vorurteilsfreiheit werden etwa manifest in seinen Beschreibungen von Sexualität. Seine Leistungsfähigkeit als reflektierter Beobachter zeigt sich dann, wenn er die eigenen Vorurteilsstrukturen durch seine direkten Wahrnehmungen bricht.

Bis in seine Spätzeit hinein beschrieb Forster sein naturgeschichtliches Forschungsverfahren als auf „Erfahrung und Vernunft" (AA, VIII: 159) beruhend: „Alles kommt [....] auf die Genauigkeit und Richtigkeit der Beobachtungen, sowie auf die unläugbare Evidenz und unfehlbare Gewissheit der Folgerungen aus dem Bemerkten an." (Ebd.: 161) Die reflektierte Beobachtung verbindet folglich 
Sinneseindruck und Vernunftschluss. Für Forsters Versuche, diese Verknüpfung zu denken, stehen die Spekulation, die Verallgemeinerung und die Schlussfolgerung. Dabei ist es stets die konkrete Erfahrung, die als Ausgangspunkt seiner Überlegungen diente und in einen größeren Zusammenhang gerückt wurde. Die Spekulation ist für ihn die schwächste, die vorsichtigste dieser Formen der Reflexion. Forster versuchte sie im Konjunktiv zu formulieren, um sie als perspektivischen Einfall darzustellen. Selbstbewusster argumentierte er hingegen, wenn er Einzelbeobachtungen verallgemeinert, was in der Regel im Rekurs auf frühere Erlebnisse oder Lektüreerfahrungen (AA, III: 344) geschieht.

Der Beobachtung muss für Forster die Interpretation folgen, müssen die „Folgerungen aus dem Bemerkten“ stehen, ein methodisches Vorgehen, das schon in seiner Vorrede zur Reise um die Welt anklingt, als er über die nur „Thatsachen“ sammelnden Gelehrten spottete (vgl. AA II: 12 f.). In der Zusammenfassung seiner Beobachtungen des Amsterdamer Hafens brachte er den konstruierenden Charakter, der seiner Beobachtungspraxis zugrunde liegt, auf den Begriff:

\footnotetext{
Dies ist mir der Totaleindruck aller dieser unendlich mannigfaltigen, zu Einem Ganzen vereinigten Gegenstände, die vereinzelt und zergliedert so klein und unbedeutend erscheinen. [...] Das Ganze ist nur dafür die Phantasie, die es aus einer gewissen Entfernung unbefangen beobachtet und die größeren Resultate mit künstlerischer Einheit begabt; die allzu große Nähe des besonderen Gegenstandes, worauf die Seele jedes Einzelnen, als auf ihren Zweck, sich konzentriert, verbürgt ihr auch des Ganzen Zusammenhang und Gestalt. (AA, IX: 300)
}

Das Ganze verdankt sich Forster allein der Einbildungskraft, dem „denkenden Auge“ des Beobachters: „Also, nicht mit dem Auge allein, sondern auch dem Verstand erscheint Amsterdam von der Wasserseite in seinem höchsten Glanze." (AA, IX: 304)

Die vergleichende Analyse steigt bei Forster zu einem akzeptierten methodologischen Verfahren auf. Die Konstanz der menschlichen Natur ist die zentrale Prämisse der grundsätzlichen Vergleichbarkeit von Völkerschaften in Raum und Zeit. Innerhalb der literarischen Gattung Reisebericht entwickelte Forster ein Verfahren des Kulturvergleichs (vgl. Garber 2003). Der reisende Naturhistoriker bezieht danach die "Stationen“ seiner Reiseroute vergleichend aufeinander und schreibt bestimmten Räumen typusbildende Eigenschaften zu. Die Kriterien derartiger Vergleiche sind die geographische Beschaffenheit der verschiedenen Weltgegenden, die Physiognomie ihrer Bewohner, deren Temperament, Fähigkeiten, Bräuche, Sprachen und ähnliches in ihren wechselseitigen Zusammenhängen. Forsters Ansatz ist so angelegt, dass diese Kriterien als zusammenhängende und daher 
vergleichbare Momente vermittelt werden. Als aufschlussreiches Beispiel lässt sich jene Beobachtung heranziehen, die Forster auf der Insel „Oster-Eyland“ im März 1774 machte:

Den wenigen Worten nach zu urtheilen, die wir von ihnen gehört hatten, dünkte uns ihre Sprache ein Dialect der Tahistischen zu seyn. Es wird also an beyden Ende der Südsee einerley Sprache geredet. Ihr ganzes Ansehen ließ uns vermuthen, daß sie ein Zweig desselbigen Volks-Stammes seyn müßten. Sie waren von mittlerer Größe, aber mager, und der Gesichtsbildung nach, den Tahitiern ähnlich, jedoch nicht so schön. (AA, II: 435)

In seinen Vergleichen zwischen den verschiedenen Völkern hob er deren Eigentümlichkeiten ebenso hervor wie ihre Ähnlichkeiten. Scharf differenzierte Forster die politischen Verhältnisse, so reichten ihm die üblichen Bezeichnungen wie Monarchie und Feudalismus nicht aus, um die spezifischen Herrschaftsverhältnisse zu kennzeichnen. Besondere Aufmerksamkeit widmete er der Rolle der Frauen in der jeweiligen Gesellschaft, sah er doch in ihrer Wertschätzung und Behandlung einen Maßstab für die Kulturstufe, auf der ein Volk steht (vgl. AA I: 276, 447 f.). Forster gelingt es schließlich, durch sein komparatistisches Verfahren die Vor- und Nachteile von Wildheits- und Zivilisationsgesellschaften zu entfalten. Dieser Vergleich zwischen Zivilisation und der „Natur" des Menschen war ein Dauerdiskussionsthema der europäischen Aufklärung.

Methodisch bewusst versuchte Forster, relevante Vergleichskriterien hierfür zu finden und aufzuzeigen, welche Elemente für die jeweiligen Strukturen charakteristisch sind, um zu gesicherten Ergebnissen $\mathrm{zu}$ gelangen. Ein überzeugender Beleg für seine Bemühungen ist sein Essay „Über Leckereien“ (1785), in dem er erörterte, welche Vergleichspunkte und -kriterien herangezogen werden können, um objektive Antworten auf aufgeworfene Fragen zu geben (Ewert 1993: 81 f.).

Vergleichen und analogisches Denken gehen bei Forster Hand in Hand. Das analogische Denken eröffnete ihm kreative kombinatorische Freiheiten. Es steht in der Tradition der scala naturae, setzt dabei Forsters sensualistische Theorie des Verstehens voraus, hebt ein erneuertes Verständnis von Induktion heraus und fordert die epistemologische Aufwertung der Wahrscheinlichkeit ein. Forster übernahm damit ein Verfahren, das zeitgenössische Philosophen wie Lambert und Mendelssohn exponiert hatten. ${ }^{15}$ Das analogische Schließen setzte Forster ein, um komplexe Phänomene auf Prädikate „einfacher" Substanzen zu reduzieren, um einen unbekannten Forschungsgegenstand auf der Grundlage von bekannten Zusammenhängen $\mathrm{zu}$ entschlüsseln und $\mathrm{zu}$ verstehen. Mit Hilfe der analogischen Heuristik werden so Forschungsge- 
genstände für die experimentelle Prüfung prospektiv zugänglich. In seinen Analogien schloss er von der Ursache auf die Wirkung, vom Inneren auf ein Äußeres und vermittelte zwischen unterschiedlichen Wissensformen wie Geschichte und Natur, Natur und Kultur. Forster begriff sein analogisches Schließen als eine konstruierende Operation in explikatorischer, epistemologischer und methodischer Hinsicht. Es hat in Forsters naturhistorisch inspiriertem Denken und Argumentieren neben seiner inventorischen auch eine judikative Funktion. Und der Autor Forster setzte die Analogie bewusst auch rhetorisch-argumentativ ein.

Bei seinem „Vergleich der verschiedenen Lebensepochen des einzelnen Menschen mit den Stufen der Kultur bei ganzen Familien und Völkern“ (AA, VII: 190) war er sich bewusst, dass eine solche Analogie zwischen individuellem organischen Wachstum und Kulturentwicklung von Zeitgenossen als überholt abgetan wurde. Forster wollte hingegen „diese Ähnlichkeit“ ausdrücklich „,wieder hervorsuchen“. Dabei war er sich der Problematik dieses Verfahrens sehr bewusst: „Ich weiß wie viel ich wage, indem ich diese Ähnlichkeit des Allgemeinen mit dem Besonderen wieder hervorsuche. Wie leicht sind nicht Ähnlichkeit überall gefunden?" (AA, VIII: 192) Im theoretischen Rahmen der Naturgeschichte ging er davon aus, dass aus der Ontogenese auch Erkenntnisse für die Phylogenese zu gewinnen sind. Aber er relativierte die Übertragung des Modells der individuellen Entwicklung auf die Menschheitsgeschichte, insofern er ihren metaphorischen Charakter hervorhob und damit den Analogieschluss als methodische Grundlage dieser Argumentation zumindest problematisierte (ebd.: 186). Forster wusste nur zu genau, dass die wahrscheinlichen Sätze der Analogie lediglich als Supplement sicheren Wissens gelten können.

Forster repräsentiert einen epistemologischen Antidogmatismus, indem er dafür plädierte, den „kleinen Geistern den armseligen Wahn (zu) überlassen, daß die Wahrheit ihnen allein eignen müsse, sie niemanden eignet - oder allen“ (AA, V: 350). Nicht nur hielt er ,eine ausschließende normalische Ansicht des Erdkreises“ für eine „Beleidigung des Verstandes“ (AA, XI: 193), sondern betonte auch seine „Überzeugung, dass jedes Bestreben, eine Vorstellung auf Kosten einer anderen herrschend $\mathrm{zu}$ machen und ihr mehr als bloß relativen Werth beizumessen, geradewegs zur Einschränkung und Lähmung unserer Geisteskräfte, so wie zur Verfinsterung unsres Verstandes führt" (AA, V: 628). Nach Forster lehrt jede hinzutretende individuelle „Verschiedenheit der Empfindungen [...] uns zu erkennen,“ dass „bloß partielle, subjektive Erkenntniß der Dinge“ (ebd.: 627) möglich ist, die sich nicht ohne 
weiteres vereinheitlichen lässt. Das gilt dann freilich auch für Forsters eigene Ansichten, die so immer nur vorläufigen, stets revidierbaren Status haben. Er setzte mit seinem epistemologisch begründeten Plädoyer für einen Pluralismus der Meinungen zum einen die Gleichheitsansprüche aller an der Wahrheitssuche Beteiligten voraus und zum anderen die räsonierende Öffentlichkeit (vgl. etwa AA, VII: 232). ${ }^{16}$

\section{Danksagung}

Den anonymen Lesern danke ich für die intensive und kritische Lektüre meiner Argumentation. Ihre anregenden Einreden sind partiell in die Überarbeitung eingeflossen.

\section{Anmerkungen}

1 Forsters Schriften werden in diesem Artikel in der Akademiewerkausgabe genutzt und im Folgenden zitiert als AA mit römischer Bandzählung.

$2 \mathrm{Zu}$ dieser geschichtstheoretischen Formel vgl. die knappe Skizze von Nolte (2002).

3 S. als Überblick Sloan (1995), Jardine/Secord/Spary (1996), Farber (2003), Müller-Wille (2009).

4 Uhlig hat in diesem Kontext zutreffend formuliert, dass es für Forsters Konzeption charakteristisch sei, ,daß es nicht mehr auf die Untersuchung isolierter Individuen ankommt, freilich auch nicht auf deren Einordnung in den bloß begrifflichen Zusammenhang eines Systems, sondern auf Kausalzusammenhänge und insbesondere die Wirkungszusammenhänge zwischen verschiedenen lebendigen Individuen" (Uhlig 1965: 51).

5 Forsters konstitutive Abneigung gegen Systeme hat die Forschung frühzeitig herausgestellt; vgl. etwa Uhlig (1965: 8), aber auch Ewert (1993).

6 Für den Artikel wurden insbesondere herangezogen AA II und III: Die Reise um die Welt; AA IV: Streitschriften und Fragmente zur Weltreise; AA V: Kleine Schriften zur Völker- und Länderkunde, AA VIII: Kleine Schriften zu Philosophie und Zeitgeschichte, AA IX: Ansichten vom Niederrhein.

7 Zu Forsters Rousseau Rezeption vgl. vor allem Kronauer (1994, 2000).

8 Zur Genese des Rassebegriffs vgl. grundlegend Sommer (1984), außerdem Boulle (2003).

9 Kronauer weist überzeugend Forsters Auseinandersetzung mit Rousseau auch für diesen Argumentationszusammenhang nach (Kronauer 1998).

10 Weiterführend zu Meiners vgl. Gierl (2008); für den weiteren theoretischen Zusammenhang vgl. Bindman (2002).

11 Vgl. AA, III: 327. Dort schreibt er an die Adresse der Klimatheoretiker: „Diejenigen Philosophen, welche den Gemütscharakter, die Sitten und das Genie der Völker lediglich vom Klima abhängen lassen, würden gewiß sehr verlegen seyn, wie sie, aus diesem allein den friedfertigen Charakter der Leute auf Neu-Caledonien erklären sollten."

12 Für den theoretischen Hintergrund vgl. vor allem Reill, Peter (2005). 
13 Vgl. dazu die problematische Interpretation von Riedel (1989).

14 Vgl. aber Rohbeck (2006, 2004), Meyer (2006).

15 Zum zeitgenössischen analogischen Denkens vgl. die anregende Studie von Rudolph (2006).

16 Vgl. dazu weiterführend die Studie von Broman (1998).

\section{Literatur}

Bernasconi, Robert (Hg.), 2006. Kant and Forster. Bristol: Thoemmes. [Bd. 3: Concepts of Race in the Eighteenth Century].

Bindman, David, 2002. Ape to Apollo. Aesthetics and the Idea of Race in the 18th Century. London: Reaktion Book.

Bödeker, Hans Erich, 2006. Die „Natur des Menschen so viel möglich in mehreres Licht... setzen“. Ethnologische Praxis bei Johann Reinhold und Georg Forster. In: Jörn Garber und Tanja Van Hoorn, Hg., Natur - Mensch - Kultur. Georg Forster im Wissenschaftsfeld seiner Zeit. Hannover: Wehrhahn, 143-170.

Bödeker, Hans Erich/Büttgen, Philippe/Espagne, Michel, Hg., 2008. Die Wissenschaft vom Menschen in Göttingen um 1800. Wissenschaftliche Praktiken, institutionelle Geographie, europäische Netzwerke. Göttingen: Vandenhoeck \& Ruprecht.

Broman, Thomas, 1998. The Habermasian Public Sphere and „Science In The Enlightenment". History of Science, 36, 123-149.

Boulle, Pierre H., 2003. Race. In: Encyclopedia of the Enlightenment. Bd. 3: MablyRuysch. Oxford: Oxford University Press, 384-387.

Chappey, Jean-Luc, 2002. La Société des Observateurs de l'Homme (1799-1804). Des anthropologues au temps de Bonaparte. Paris: Sociéte des Études Robespierre.

Corbey, Raymond/Theunissen, Bert, Hg., 1995. Ape, Man, Apeman. Views since 1600. Leiden: Department of Prehistory, Leiden: Leiden University.

Dougherty, Frank William Peter, 1996. Buffons Bedeutung für die Entwicklung des anthropologischen Denkens im Deutschland der zweiten Hälfte des 18. Jahrhunderts. In: Ders., Hg., Gesammelte Aufsätze zu Themen der klassischen Periode der Naturgeschichte. Göttingen: Klatt, 70-88.

Dreitzel, Horst, 1981. Die Entwicklung der Historie zur Wissenschaft. Zeitschrift für historische Forschung, 8, 257-284.

Ewert, Michael, 1993. „Vernunft, Gefühl und Phantasie, im schönsten Tanz vereint“. Die Essayistik Georg Forsters. Würzburg: Königshausen \& Neumann.

Farber, Paul Lawrence, 2003. Natural History. In: Encyclopedia of the Enlightenment. Bd. 3: Mably-Ruysch. Oxford: Oxford University Press, 124-130.

Ferguson, Adam, 1772. Grundsätze der Moralphilosophie. Übers. v. Christian Garve. Leipzig: Dyck.

Fink, Gonthier-Louis, 1987. Von Winckelmann bis Herder. Die deutsche Klimatheorie in europäischer Perspektive. In: Gerhard Sauder, Hg., Johann Gottfried Herder 1744-1803. Hamburg: Meiner, 156-176.

Forster, Georg, 1958f. Werke. Sämtliche Schriften, Tagebücher, Briefe, hrsg. v. d. Deutschen Akademie der Wissenschaften zu Berlin, 20 (bisher 19) Bände. Berlin: Akademie-Verlag.

Garber, Jörn, 1992. Von der Menschheitsgeschichte zur Kulturgeschichte. Zum geschichtstheoretischen Kulturbegriff der deutschen Spätaufkärung. In: Ders., Hg., Spätabsolutismus und bürgerliche Gesellschaft. Studien zur deutschen Staats- und Gesellschaftstheorie im Übergang zur Moderne. Frankfurt a. M.: Keip, 409-433.

Garber, Jörn, 1994. Anthropologie und Geschichte. Spätaufklärerische Staats- und Geschichtsdeutung im Metaphernfeld von Mechanismus und Organismus. In: 
Claus-Volker Klenke, Jörn Garber und Dieter Heintze, Hg., Georg Forster in interdisziplinärer Perspektive. Berlin: Akademie-Verlag, 193-210.

Garber, Jörn, 1995. Von der „Geschichte des Menschen“ zur „Geschichte der Menschheit . Anthropologie, Pädagogik und Zivilisationstheorie in der deutschen Spätaufklärung. Jahrbuch für Historische Bildungsforschung, 5, 31-54.

Garber, Jörn, 1999. Selbstreferenz und Objektivität. Organisationsmodelle von Menschheit- und Weltgeschichten in der deutschen Spätaufklärung. In: Hans Erich Bödeker, Peter H. Reill, und Jürgen Schlumbohm, Hg., Wissenschaft als kulturelle Praxis. 1750-1900. Göttingen: Vandenhoeck \& Ruprecht, 137-186.

Garber, Jörn, 2003. „Wahrheit ist das Verhältnis der Dinge unter einander und zu uns“. Empirismus - Konstruktion - „Gedankenbild“ (Georg Forster: 17541794). In: Barbara Friebertshäuser und Annedore Prengel, Hg., Handbuch Qualitative Forschungsmethoden in der Erziehungswissenschaft. Weinheim/ München: Juventa-Verlag, 221-244.

Gierl, Martin, 2008. Christoph Meiners' Geschichte der Menschheit und Göttinger Universalgeschichte. Rasse und Nation als Politisierung der deutschen Aufklärung. In: Hans Erich Bödeker, Philippe Büttgen und Michel Espagne, Hg., Die Wissenschaft vom Menschen in Göttingen um 1800. Wissenschaftliche Praktiken, institutionelle Geographie, europäische Netzwerke. Göttingen: Vandenhoeck \& Ruprecht, 419-433.

van Hoorn, Tanja, 2003. Physische Anthropologie und normative Ästhetik. Georg Forsters kritische Rezeption der Klimatheorie in seiner "Reise um die Welt". Georg Forster Studien, 8, 139-162.

van Hoorn, Tanja, 2004. Dem Leibe abgelesen. Georg Forster im Kontext der physischen Anthropologie des 18. Jahrhunderts. Tübingen: Niemeyer.

van Hoorn, Tanja, 2006. Das anthropologische Feld der Aufklärung. Ein heuristisches Modell und ein exemplarischer Situierungsversuch. In: Jörn Garber und Tanja Van Hoorn, Hg., Natur - Mensch - Kultur. Georg Forster im Wissenschaftsfeld seiner Zeit. Hannover: Wehrhahn, 125-141.

Jahn, Ilse, 1994. „Scientia Naturæ - Naturbetrachtung oder Naturwissenschaft?“ Georg Forsters Erkenntnisfragen zu biologischen Phänomenen in VorlesungsManuskripten aus Wilna und Mainz (1786-1793). In: Claus-Volker Klenke, Jörn Garber und Dieter Heintze, Hg., Georg Forster in interdisziplinärer Perspektive. Berlin: Akademie-Verlag, 159-176.

Jardine, Nicholas/Secord, James A./Spary, Emma C., Hg., 1996. Cultures of Natural History. Cambridge: Cambridge University Press.

Kant, Immanuel, 1785. Bestimmung des Begrifs einer Menschenrace. Berlinische Monatsschrift, 6, 390-417.

Kant, Immanuel, 1957 [1790]. Kritik der Urteilskraft. In: Ders., Werke, hg. v. Wilhelm Weischedel, Bd. 9. Wiesbaden: Insel-Verlag, 237-622.

Kohl, Karl-Heinz, 1981. Entzauberter Blick. Das Bild vom Guten Wilden und die Erfahrung der Zivilisation. Berlin: Medusa.

Kondylis, Panajotis, 1986. Die Aufklärung im Rahmen des neuzeitlichen Rationalismus. München: Dt. Taschenbuch-Verlag.

Kronauer, Ulrich, 1994. Rousseaus Kulturkritik aus der Sicht Georg Forsters. In: Claus-Volker Klenke, Jörn Garber und Dieter Heintze, Hg., Georg Forster in interdisziplinärer Perspektive. Berlin: Akademie-Verlag, 147-156.

Kronauer, Ulrich, 1998. Zurück zu den Affen, oder über die natürliche Güte des Menschen. Rousseaus Kulturkritik und die Folgen. Georg Forster Studien, 2, 79-107.

Kronauer, Ulrich, 2000. Georg Forsters Einleitung zu "Cook der Entdecker“. Forsters Auseinandersetzung mit Rousseau über Fortschritt und Naturzustand. In: Jörn Garber, Hg., Wahrnehmung - Konstruktion - Text. Tübingen: Niemeyer, 31-42.

Medick, Hans, 1973. Naturzustand und Naturgeschichte der bürgerlichen Gesellschaft. Die Ursprünge der bürgerlichen Sozialtheorie als Geschichtsphilosophie und als Sozialwissenschaft bei Samuel Pufendorf, John Locke uns Adam Smith. Göttingen: Vandenhoeck \& Ruprecht. 
Meiners, Christoph, 1785. Grundriß der Geschichte der Menschheit. Lemgo: Meyer. Meyer, Annette, 2006. Von der "Science of Man“ zur "Naturgeschichte der Menschheit". Einflüsse angelsächsischer Wissenschaft im Werk Georg Forsters. In: Jörn Garber und Tanja Van Hoorn, Hg., Natur - Mensch - Kultur. Georg Forster im Wissenschaftsfeld seiner Zeit. Hannover: Wehrhahn, 29-52.

Meyer, Annette, 2008. Von der Wahrheit zur Wahrscheinlichkeit. Die Wissenschaft vom Menschen in der schottischen und deutschen Aufklärung. Tübingen: Niemeyer.

Moravia, Sergio, 1970. La Scienza dell' Uomo nell Settecento. Bari: Laterza.

Moravia, Sergio, 1982. Filosofia e Scienze Umane nell' Età dei Lumi. Firenze: Sansoni.

Müller-Wille, Staffan, 2009. Naturgeschichte. In: Friedrich Jäger, Hg., Enzyklopädie der Neuzeit, Bd. 8. Stuttgart/Weimar: Metzler, Sp. 1175-1196.

Mulsow, Martin, 1995. Selbsterhaltung. In: Historisches Wörterbuch der Philosophie, Bd 9. Basel: Schwabe, Sp. 393-406.

Nolte, Paul, 2002. Gleichzeitigkeit des Ungleichzeitigen. In: Stefan Jordan, Hg., Lexikon Geschichtswissenschaft. Hundert Grundbegriffe. Stuttgart: Philipp Reclam, 134-137.

Nowitzki, Hans-Peter, 2006. "Schön ist das Schauspiel ringender Kräfte“. „Lebenskräfte“ bei Christoph Wilhelm Hufeland, Johann Gottfried Herder und Georg Forster. Jahrbuch für Europäische Wissenschaftskultur, 2, 209-246.

Oz-Salzberger, Fania, 1995. Translating the Enlightenment. Scottish Civic Discourse in Eighteenth-Century Germany. Oxford: Clarendon Press.

Proß, Wolfgang, 1999. Die Begründung der Geschichte aus der Natur: Herders Konzept von "Gesetzen“ in der Geschichte. In: Bödeker, Hans Erich/Reill, Peter H./Schlumbohm, Jürgen, Hg., Wissenschaft als kulturelle Praxis. 17501900. Göttingen: Vandenhoeck \& Ruprecht, 187-226.

Reill, Peter Hanns, 2005. Vitalizing Nature in the Enlightenment. Berkeley/Los Angeles/London: University of California Press.

Rennie, Niel, 1995. Far-Fetched Facts. The Literature of Travel and the Idea of the South Seas. Oxford: Clarendon Press.

Riedel, Manfred, 1989. Historismus und Kritizismus. Kants Streit mit G. Forster und J.G. Herder. In: Ders., Urteilskraft und Vernunft. Kants ursprüngliche Fragestellung, Frankfurt a. M.: Suhrkamp, 148-169.

Roger, Jaques, 1989. Buffon. Un philosophe au Jardin du Roi. Paris: Fayard.

Rohbeck, Johannes, 2004. Historisierung des Menschen. Zum Verhältnis von Naturgeschichte und Geschichtsphilosophie. In: Walter Schmitz und Carsten Zelle, Hg., Innovation und Transfer - Naturwissenschaft, Anthropologie und Literatur im 18. Jahrhundert. Dresden: Thelem, 121-130.

Rohbeck, Johannes, 2006. Naturgeschichte und Geschichte der Menschheit. Georg Forsters Geschichtsphilosophie im Kontext der europäischen Aufklärung. In: Jörn Garber und Tanja Van Hoorn, Hg., Natur - Mensch - Kultur. Georg Forster im Wissenschaftsfeld seiner Zeit. Hannover: Wehrhahn, 13-28.

Rudolph, André, 2006. Figuren der Ähnlichkeit. Johann Georg Hammans Analogiedenken im Kontext des 18. Jahrhunderts. Tübingen: Max Niemeyer Verlag.

Sloan, Phillip, 1995. The Gaze of Natural History. In: Christopher Fox, Roy Porter und Robert Wokler, Hg., Inventing Human Science. Eighteenth-Century Domains. Berkeley u. a.: University of California Press, 112-151.

Soemmering, Samuel Thomas, 1785. Ueber die körperliche Verschiedenheit des Negers vom Europäer. Franfurt/Mainz: Varrentrapp Sohn und Wenner.

Sommer, Antje, 1984. Rasse. In: Otto Brunner, Werner Conze und Reinhart Koselleck, Hg., Geschichtliche Grundbegriffe. Historisches Lexikon zur politisch-sozialen Sprache in Deutschland, Bd. 5. Stuttgart: Klett-Cotta, 137-146.

Stumman-Bower, Ruth, 2004. Georg Forster. Übersetzer, Herausgeber und Rezensent am Beispiel der Nachrichten von den Pelow-Inseln in der Westgegend des Stillen Ozeans (1789). Georg Forster Studien, 9, 181-223.

Toulmin, Stephan/Goodfield, June, 1985. Entdeckung der Zeit. Frankfurt a. M.: Fischer, 157-158. 
Georg Forsters Entwurf einer „Wissenschaft vom Menschen”

Uhlig, Ludwig, 1965. Georg Forster. Einheit und Mannigfaltigkeit in seiner geistigen Welt. Tübingen: Niemeyer.

Uhlig, Ludwig, 2003. Theoretical or Conjectural History. Georg Forsters „Voyage Round the World" im zeitgenössischen Kontext. Germanisch-Romanische Monatsschrift, 53, 399-414.

Uhlig, Ludwig, 2004. Georg Forster. Lebensabenteuer eines gelehrten Weltbürgers (1754-1794). Göttingen: Vandenhoek \& Ruprecht.

Wyder, Margrit, 1998. Goethes Naturmodell. Die Scala Naturae und ihre Tranformationen. Köln u. a.: Böhlau.

\section{Hans Erich Bödeker}

Thomas-Dehler-Weg 16

D-37075 Göttingen, Deutschland

E-Mail: hansboedeker@web.de 